\title{
BMJ Open Variation in relationships between maternal age at first birth and pregnancy outcomes by maternal race: $a$ population-based cohort study in the United States
}

\author{
Laura Schummers (1) , ${ }^{1}$ Michele R Hacker, ${ }^{2,3}$ Paige L Williams, ${ }^{3,4}$ \\ Jennifer A Hutcheon, ${ }^{5}$ Tyler J Vanderweele, ${ }^{3,4}$ Thomas F McElrath, ${ }^{6}$ \\ Sonia Hernandez-Diaz ${ }^{3}$
}

To cite: Schummers L, Hacker MR, Williams PL, et al. Variation in relationships between maternal age at first birth and pregnancy outcomes by maternal race: a population-based cohort study in the United States. BMJ Open 2019;9:e033697. doi:10.1136/ bmjopen-2019-033697

- Prepublication history and additional material for this paper are available online. To view these files, please visit the journal online (http://dx.doi. org/10.1136/bmjopen-2019033697).

Findings were presented as a poster at the 51 st Annual Meeting of the Society for Epidemiologic Research (19-22 June 2018) in Baltimore, MD, and at the 31 st Annual Meeting of the Society for Perinatal Epidemiologic Research (18-19 June 2018) in Baltimore, MD.

Received 16 August 2019 Revised 07 November 2019 Accepted 20 November 2019

Check for updates

(C) Author(s) (or their employer(s)) 2019. Re-use permitted under CC BY-NC. No commercial re-use. See rights and permissions. Published by BMJ.

For numbered affiliations see end of article.

Correspondence to Dr Laura Schummers; laura.schummers@ubc.ca

\section{ABSTRACT}

Objective To estimate absolute risks of obstetric outcomes in the USA according to maternal age at first birth from age 15 to 45 separately by maternal race. Design and setting Population-based cohort study. Setting Vital statistics Birth Cohort-Linked Birth- Infant Death Data Files and Fetal Death Data Files in the USA. Participants 16514849 births to nulliparous women from 2004 to 2013.

Outcome measures We estimated absolute risks of obstetric outcomes (multiple gestations, caesarean delivery, early and late preterm birth, small for gestational age birth, stillbirth, neonatal mortality, postneonatal infant mortality) at each year of maternal age from 15 to 45 years using logistic regression in the overall population and stratified by maternal race. We modelled maternal age flexibly to allow curvilinear shapes and plotted risk curves for each outcome.

Results In the overall population, multiple gestations, caesarean delivery and stillbirth risks were lowest at young maternal ages with linear or quadratic increases with age. Curves for preterm birth, small for gestational age, neonatal mortality and postneonatal mortality were $u$ or $j$ shaped, with nadirs between 20 and 29 years, and elevated risks at both younger and older maternal ages. In race-stratified analyses, the shapes of the curves were generally similar across races. Risks increased for all women for all outcomes after age 30 . However, increased risks at young maternal ages were most pronounced for white and Asian/Pacific Islander women, for whom young childbearing was least common. Conversely, risks at older ages were more pronounced for Black and American Indian/Alaska Native women, for whom delayed childbearing was least common.

Conclusion Our findings confirm risks associated with first births to women younger than 20 and older than 30 years, provide easily interpretable risk curves and illuminate variability in these relationships across categories of maternal race in the USA.
Strengths and limitations of this study

- Population-based design with large sample size enables examination of rare outcomes separately by racial group.

- Stratified risk curves enable detection of variability in age-outcome relationships according to maternal race.

- Year-by-year absolute risks are easy to understand and interpret for clinicians and childbearing women and families.

- Data set lacks clinical detail necessary to examine some important sociodemographic variables (eg, education, income) and clinically detailed outcomes (eg, hypertensive disorders of pregnancy).

- Birth cohort restricted to pregnancies continuing to 20 weeks of gestation or longer, which cannot account for infertility or miscarriage risks, which both increase with increasing maternal age.

\section{INTRODUCTION}

Adverse obstetric outcomes, including preterm birth, stillbirth and infant mortality, are more common among births to women older than $35^{1-7}$ and younger than $20^{89}$ compared with births to women 20-29 years of age. Despite considerable research examining the risks associated with advanced (eg, 35 years or older) or young (eg, younger than 20) maternal age, information about agespecific absolute risks of adverse pregnancy and birth outcomes in a US population is scarce.

Maternal age at first birth varies by racial group in the USA, with the youngest ages for American Indian/Alaska Native women, and oldest ages for Asian/Pacific Islander women. ${ }^{10}$ Risks of adverse pregnancy and birth outcomes, including caesarean delivery, 
preterm birth and infant mortality, also vary by racial group in the USA. ${ }^{11-15}$ Both Black women and American Indian/Alaska Native women have the highest risks of adverse birth outcomes, which has been attributed to racism, racial discrimination and related psychosocial stress. ${ }^{16}$ Moreover, different distributions of age-related risk factors for adverse pregnancy outcomes by racial group (eg, socioeconomic factors, pre-pregnancy health status, access to antenatal care) may result in different relationships between maternal age and pregnancy risks by race. Differences in the relationships between maternal age at first birth and absolute risks of pregnancy outcomes according to maternal race are not well understood.

This paper presents absolute risks of birth outcomes in the USA according to maternal age at first birth, allowing for curvilinear relationships. Age-specific risk curves are presented for the overall population and separately for each racial group.

\section{MATERIALS AND METHODS}

Using Birth Cohort-Linked Birth - Infant Death Data Files and Fetal Death Data Files available through the National Center for Health Statistics, our study population included all births to nulliparous women in the USA from 2004 to 2013. Fetal deaths before 20 completed weeks of gestation or with birth weights less than $350 \mathrm{~g}$ were excluded. Multiple gestation pregnancies and pregnancies with congenital anomalies or structural defects were retained in the study population.

We examined those outcomes with established validity in this data set $^{17-19}$ : multiple gestation pregnancies, caesarean delivery, early preterm birth (live birth before 32 completed weeks of gestation, according to the obstetric/clinical gestational age estimate),${ }^{20}$ late preterm birth (live birth after 32 but before 37 completed weeks of gestation according to the obstetric/clinical gestational age estimate), small for gestational age (SGA, $<10$ th percentile for sex and gestational age according to the same population-level growth charts for all racial groups, among singleton births), ${ }^{21}$ stillbirth (fetal death at $\geq 20$ weeks of gestation or birth weight equal to or greater than $350 \mathrm{~g}$ ), neonatal mortality (death in the first 28 days of life) and postneonatal infant mortality (death between 28 and 364 days of age).

We examined the relationship between maternal age at first birth and each outcome using logistic regression models. We modelled maternal age flexibly as a continuous variable using restricted cubic splines to allow curvilinear shapes, with four knots placed at the 5th, 35th, 65 th and 95th percentiles of maternal age in the study population (ages 17, 22, 28 and 36). ${ }^{22}$ We used bootstrapping to estimate CIs to account for non-independence between outcomes of multiple gestation births, and drew 50 samples with replacement from the study population. ${ }^{23}$ We used logistic regression estimates to calculate absolute risks of each outcome at each maternal age from 15 to 45 years. ${ }^{24}$ These unadjusted estimates represent population average risks at each maternal age, including different distributions of age-related risk factors at each age (eg, more infertility treatments and multiple gestations in older women and lower socioeconomic status among younger women). We then estimated risks adjusted for those variables that are associated with maternal age and pregnancy outcomes and are measured accurately in these data: marital status, inadequate prenatal care (fewer than five prenatal care visits) and indicator variables for calendar year with 2013 as the reference year. Fetal Death Data Files do not have information on number of antenatal visits, so models for stillbirth were not adjusted for inadequate prenatal care.

To examine the potential variability of the relationship between maternal age at first birth and outcomes by maternal race, we estimated age-specific absolute risks separately for each maternal racial group as identified on the birth certificate (white, Black, Alaska Native/American Indian, Asian/Pacific Islander).

We graphed the predicted risks at each year of maternal age at first birth to illustrate the shape of the risk curve for the full maternal age spectrum examined in the overall population and separately by maternal racial group. We then estimated risk differences comparing predicted risks at young (15 and 20 years) and advanced (30, 35, 40 and 45 years) maternal ages with predicted risks at age 25 for the whole population and separately for each maternal racial group, with $95 \%$ CIs calculated based on predicted risks. ${ }^{25}$

\section{Sensitivity analyses}

In a sensitivity analysis to examine the joint role of maternal race and Hispanic ethnicity, we repeated stratified analyses using the following categories: non-Hispanic white, non-Hispanic Black, non-Hispanic other race, and Hispanic. We conducted a sensitivity analysis in which we graphed and tabulated the observed proportion of the population at each maternal age and compared estimates obtained from modelling each outcome using restricted cubic spline transformed maternal age. To examine whether observations with imputed maternal age, those in which recorded maternal age differed from reported maternal age, and those with imputed maternal race impacted our estimates, we conducted a sensitivity analysis excluding these records and compared estimates with those from the whole cohort. We used Stata V.14.2 to conduct all analyses. ${ }^{26}$

\section{Patient and public involvement}

Patients were not involved with this study, nor were members of the public.

\section{RESULTS}

Of 41339104 births at $\geq 20$ weeks of gestation or birth weight $\geq 350 \mathrm{~g}$ in the USA from 2004 to 2013, our cohort included 16514849 births to nulliparous women. Mean age at first birth increased from 25.2 to 26.0 years over 
Table 1 Demographic and clinical characteristics of all births to nulliparous women in the United States according to maternal age at first birth, 2004-2013

\begin{tabular}{|c|c|c|c|c|c|c|c|c|}
\hline & Age $<20$ & Ages 20-24 & Ages 25-29 & Ages 30-34 & Ages 35-39 & $\begin{array}{l}\text { Ages } \\
40-44\end{array}$ & Age $\geq 45$ & Total \\
\hline & 3165587 & 4919066 & 4232285 & 2834401 & 1105766 & 237837 & 19907 & 16514849 \\
\hline \multicolumn{9}{|l|}{ Maternal race } \\
\hline White & 71.3 & 76.4 & 79.9 & 78.6 & 77.8 & 77.6 & 76.6 & 76.8 \\
\hline Black & 25.0 & 18.7 & 10.4 & 8.4 & 9.4 & 10.9 & 11.4 & 15.3 \\
\hline $\begin{array}{l}\text { American Indian/Alaska } \\
\text { Native }\end{array}$ & 1.9 & 1.3 & 0.6 & 0.4 & 0.4 & 0.3 & 0.3 & 1.0 \\
\hline Asian/Pacific Islander & 1.8 & 3.6 & 9.0 & 12.6 & 12.4 & 11.2 & 11.7 & 6.9 \\
\hline Hispanic ethnicity & 32.4 & 24.6 & 16.3 & 13.1 & 13.1 & 12.6 & 10.1 & 21.0 \\
\hline Unmarried & 87.7 & 62.5 & 26.6 & 15.9 & 18 & 23 & 21.6 & 46.5 \\
\hline Missing paternal information & 12.3 & 6.9 & 2.7 & 1.7 & 2.3 & 3.3 & 3.8 & 5.6 \\
\hline Inadequate prenatal care & 6.7 & 4.2 & 2.6 & 2.1 & 2.3 & 2.6 & 2.8 & 3.8 \\
\hline
\end{tabular}

Cells show column percentages.

Maternal age was imputed in 463773 (2.8\%) observations. Maternal race was imputed in 704767 (4.3\%) observations. Hispanic ethnicity was missing in $100589(0.8 \%)$ observations.

Previous pregnancy ending in spontaneous or induced abortion was missing in $124814(0.5 \%)$ observations.

Inadequate prenatal care defined as fewer than five visits. Number of prenatal visits was missing in 508876 (3.2\%) observations, as well as all fetal death records $(n=122254 ; 0.8 \%)$. Fetal death records were excluded from the denominator for this variable.

Prior spontaneous or therapeutic abortion defined as total birth order $>1$ in a nulliparous woman. Total birth order was missing in 70906 (0.4\%) observations.

the study period, caused by a pronounced decrease in the proportion of births to women younger than $20(20.6 \%$ to $14.7 \%$ ), and a small increase in births to women older than 40 (from $1.5 \%$ to $1.7 \%$ ). The distribution of sociodemographic characteristics varied by maternal age, as shown in table 1 . Women younger than 20 were most likely to be unmarried at the time of first birth $(87.5 \%)$, followed by those aged 20-25 $(62.3 \%)$. Excluding the $3.2 \%$ of women with missing prenatal care data, inadequate prenatal care was uncommon across all age groups (3.8\% of all births), though women younger than age 20 were more likely to receive inadequate prenatal care $(6.7 \%)$.

The distribution of maternal age at first birth varied markedly by racial group, as shown in figure 1. Age distributions were similar among Black and Alaska Native/ American Indian women, with means of 23.1 and 22.1 years, respectively. The distribution for white women was shifted to older ages (mean 25.5 with bimodal peaks near ages 20 and 30), and to substantially older ages for Asian/Pacific Islander women (mean 28.9). Asian/ Pacific Islander women were most likely to be married ( $18.4 \%$ unmarried), followed by white women $(42.1 \%$ unmarried), while the majority of Black and Alaska Native/American Indian women were unmarried (78.7\% and $73.8 \%$ ). Alaska Native/American Indian women were least likely to have adequate prenatal care $(7.3 \%$ inadequate), while Asian/Pacific Islander women were most likely ( $3.2 \%$ inadequate).

The overall incidence of adverse birth outcomes was highest for caesarean delivery $(31.4 \%)$, moderate for late preterm birth and SGA $(7.9 \%$ and $5.8 \%$, respectively) and low for other adverse birth outcomes $(0.2 \%-2.1 \%)$. Unadjusted and adjusted curves showing risks for each outcome at each maternal age from 15 to 45 years in the overall population are displayed in figure 2. Unadjusted risks of multiple gestations, caesarean delivery and stillbirth increased progressively with increasing maternal age. For all other outcomes, nadirs occurred between ages 25 and 30, after which risks increased continuously with increasing maternal age. Risks of early and late preterm births, SGA, neonatal mortality and postneonatal infant mortality also were elevated among women younger than

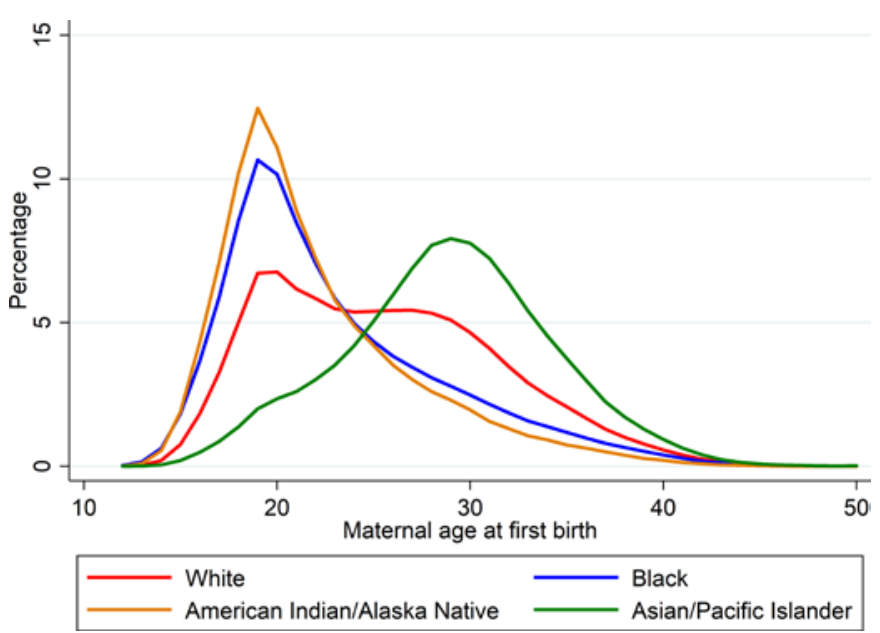

Figure 1 Distribution of maternal age at first birth by maternal race in the USA 2004-2013. 

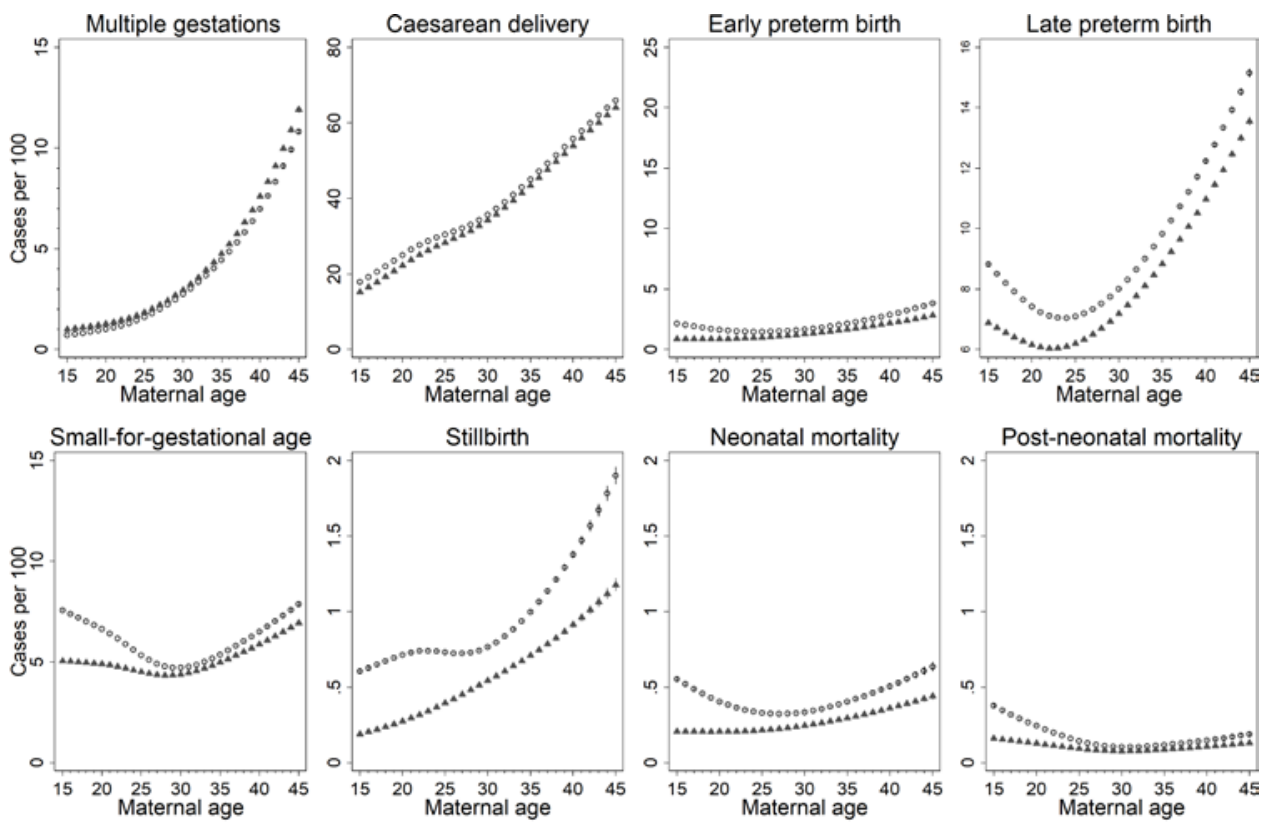

○ Unadjusted Predicted Absolute Risk

$95 \% \mathrm{Cl}$

$\Delta$ Adjusted Absolute Risk

$95 \% \mathrm{Cl}$

Figure 2 Adjusted and unadjusted absolute risks of adverse pregnancy and birth outcomes according to maternal age at first birth among all births to nulliparous women in the USA 2004-2013.

20-25 in unadjusted models. While stillbirth risk remained low until age 30 and was highest among older women, postneonatal infant mortality risks were highest among women younger than 25 and only slightly elevated after age 35. Adjustment for covariates did not substantially change estimated risks for multiple gestations, caesarean delivery or late preterm birth. After adjustment, elevated risks at maternal ages younger than 25 were substantially attenuated for SGA and postneonatal mortality and were no longer present for early preterm birth and neonatal mortality. Adjustment did not substantially attenuate increased risks observed for maternal age older than 25 for any outcome.

Risk curves stratified by maternal race are shown in figure 3 (unadjusted) and in figure 4 (adjusted). Relationships between maternal age and multiple gestations
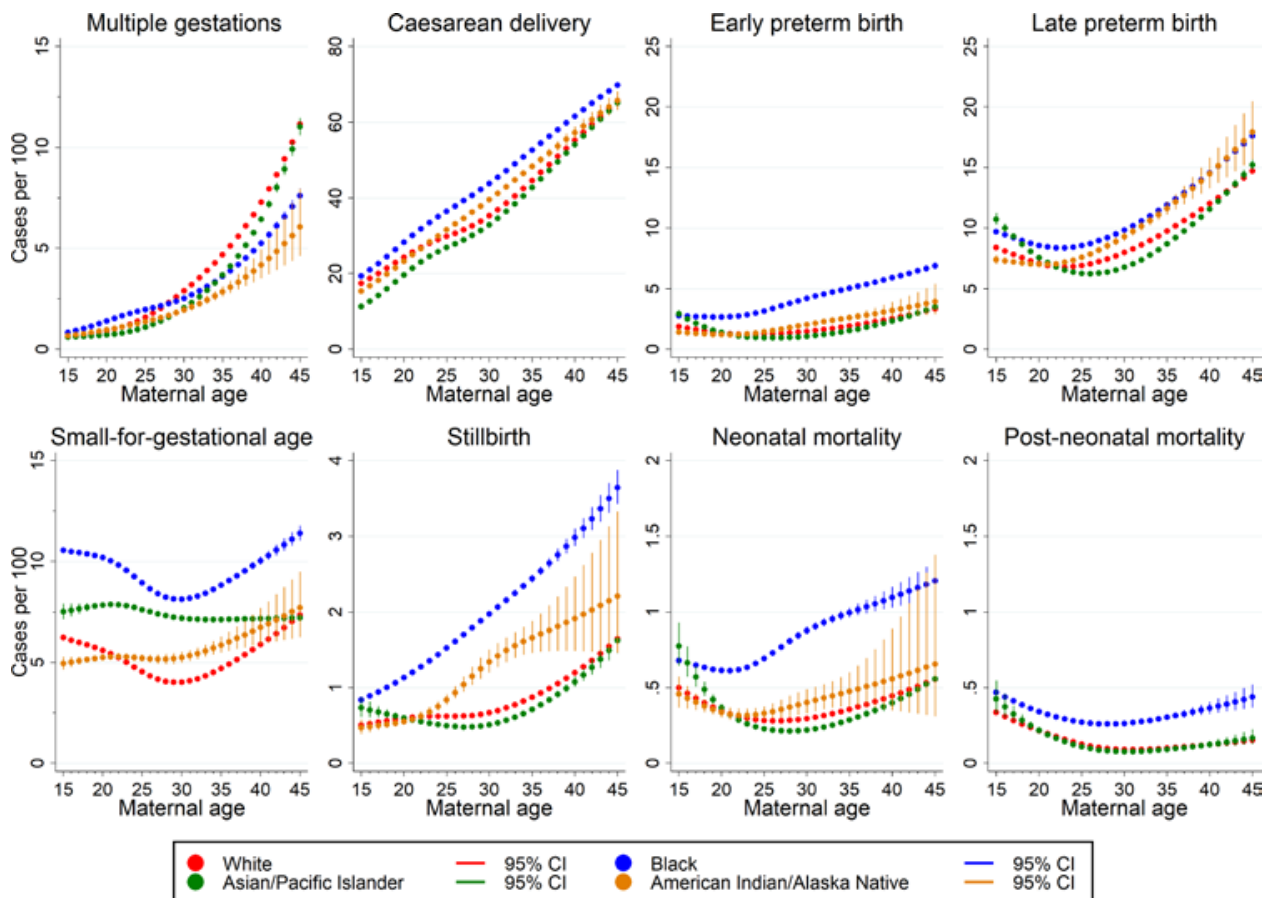

Figure 3 Unadjusted absolute risks of obstetric outcomes according to maternal age at first birth among all births to nulliparous women in the USA 2004-2013 stratified by maternal race. 

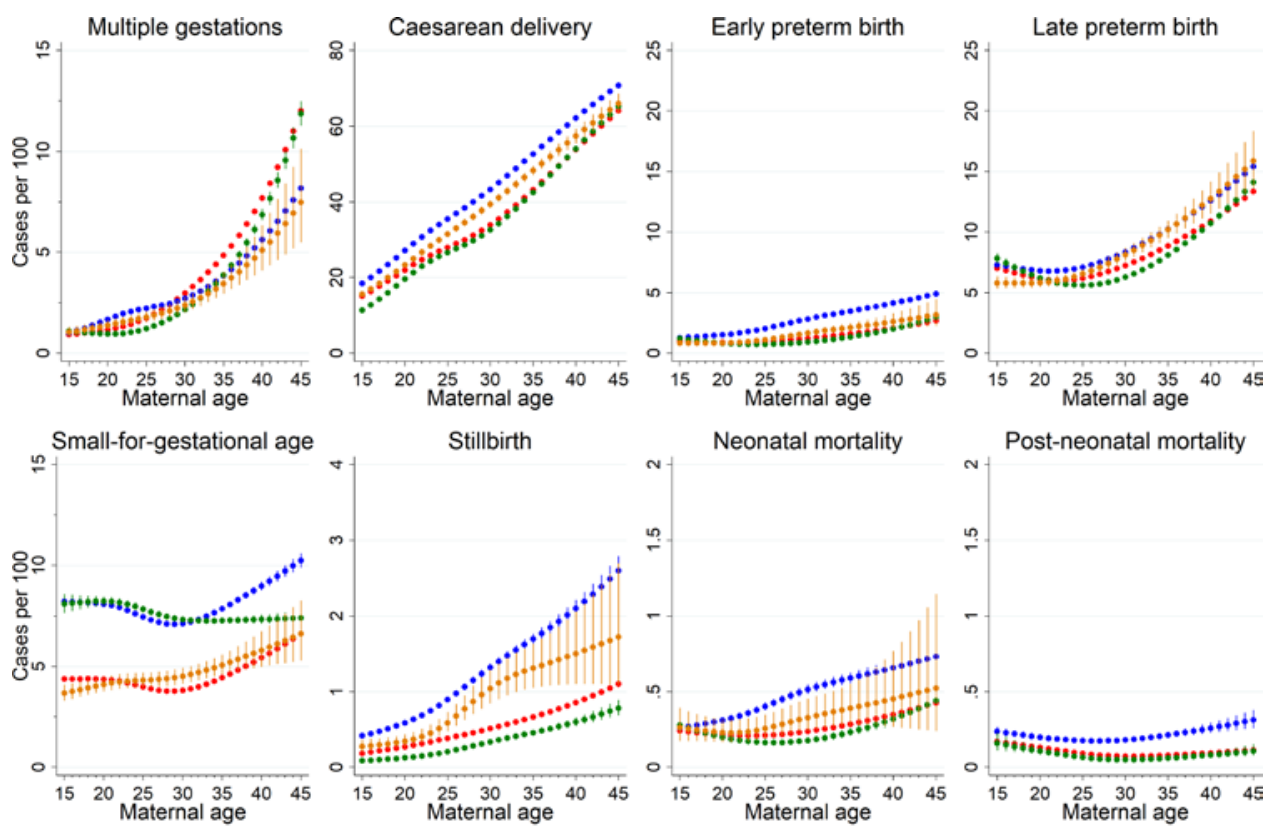

$$
\begin{aligned}
& \text { White } \\
& \text { Asian/Pacific Islander } \quad-95 \% \mathrm{Cl} \text { Cl } 8 \text { Back } \\
& \text { American Indian/Alaska Native }=95 \% \mathrm{Cl}
\end{aligned}
$$

Figure 4 Adjusted absolute risks of obstetric outcomes according to maternal age at first birth among all births to nulliparous women in the USA 2004-2013 stratified by maternal race.

and caesarean delivery risk were similar across racial groups, though multiple gestations increased more rapidly with maternal age for white and Asian/Pacific Islander women. While early and late preterm birth risks were elevated at young maternal ages for Asian/Pacific Islander and white women, risks were lower at young ages for Black and Alaska Native/American Indian women. Early preterm birth risks increased for all groups after age 25 , but increased almost twice as quickly among Black women, while late preterm birth risks increased more rapidly with maternal age for Alaska Native/American Indian women. Among Asian/Pacific Islander women, SGA risk was inversely associated with maternal age, while risks increased with increasing maternal age for Alaska Native/American Indian women.

The relationships between SGA risk and maternal age were $\mathrm{u}$ shaped for both white and Black women, with lowest risks for white women and highest risks for Black women across the age spectrum. For white and Alaska Native/American Indian women, stillbirth risks were low from age 15 to 20 , then increased with age, while for Asian/Pacific Islander women, risks were slightly elevated until age 20 , lowest at 25 and increased with age after age 25. In contrast, stillbirth risks did not have a stable plateau for Black women at any age, but rather increased linearly with maternal age from age 15 to 45 . For Asian/Pacific Islander and white women, neonatal risk curves were $\mathrm{u}$ shaped with nadirs between ages 25 and 30, although increased risks at young maternal age (before 25) were most pronounced for Asian/Pacific Islander women. Conversely, among Black women, neonatal mortality risks were lowest at age 20, and increased rapidly with maternal age after age 20. For Asian/Pacific Islander and white women, postneonatal mortality risks were substantially increased at young maternal ages, decreased to nadirs between ages 25 and 30 and increased very gradually with increasing age after 30. For Black women, postneonatal mortality risks were $\mathrm{u}$ shaped with a nadir at age 30. Beyond differences in curve shapes for Black women compared with women from other racial groups, Black women had markedly higher risks of early preterm birth, SGA, stillbirth and neonatal mortality across the age spectrum than women from all other racial groups. Due to fewer than 500 cases of postneonatal mortality among Alaska Native/American Indian women, risk estimates for this group were unstable and are not presented. Adjusted risks were similar to unadjusted risks for multiple gestations and caesarean delivery for all racial groups. Risks of all other outcomes were lower after adjustment for all racial groups from age 15 to 45 . Increased risks of early preterm delivery, stillbirth and neonatal mortality at young maternal ages were substantially attenuated by adjustment, particularly for Asian/Pacific Islander and white women. Adjustment also attenuated the slope of increasing risk associated with increasing maternal age for Black and Native American/Alaska Native women for stillbirth and neonatal mortality.

Complementing risk curves presented in the figures, predicted risks tabulated at 5-year increments of maternal age are presented in online supplementary table 1 and risk differences comparing risks at young (15 and 20 years) and advanced (30, 35, 40 and 45 years) maternal ages with risks at age 25 are presented in online supplementary table 2 , for the overall population and stratified by maternal race. 
In our sensitivity analysis jointly examining maternal race and ethnicity, we found similar distributions of age at first birth among non-Hispanic white women and Hispanic women of all races. The bimodal age distribution among white women was less pronounced after excluding Hispanic white women, indicating that the presence of two underlying populations of white women with different age was largely explained by Hispanic ethnicity (online supplementary figure 1 ). As shown in online supplementary figures 2 and 3, risks for Hispanic women were somewhat higher than for white women, but generally followed similar shapes. After excluding Hispanic women from other groups, risks were lower for non-Hispanic white women and risks were similar for non-Hispanic Black women as when Hispanic Black women were included in this group. Risks for those in the non-Hispanic 'other race' group combined the heterogenous risk curves of Asian/Pacific Islander and American Indian/Alaska Native women.

When we plotted observed risks without modelling (proportion with each outcome at each maternal age, online supplementary figure 4), curves were similar to those based on our models at most maternal ages, although the shapes were less smooth in the extremes of maternal age (particularly near age 45). Likewise, estimates from the sensitivity analysis excluding observations with imputed maternal age $(186933 ; 1.4 \%)$ or maternal race $(48051 ; 0.8 \%)$, and those in which recorded maternal age differed from reported maternal age (446 701; 3.3\%), did not differ from estimates from the whole cohort. We thus retained observations with imputed maternal age or race in our analyses.

\section{DISCUSSION}

This paper presents a detailed examination of the relationship between maternal age at first birth and absolute risks of major pregnancy and birth outcomes. We found elevated risks for all outcomes except postneonatal infant mortality among women older than 30, and SGA, early and late preterm births, neonatal mortality and postneonatal mortality among women younger than 20 . Nadirs for multiple gestations, caesarean delivery and stillbirth occurred at maternal ages younger than 20, while nadirs for all other outcomes were between ages 25 and 30 for most racial groups. Our findings were consistent with previous examinations of maternal age using a categorical approach in the USA, ${ }^{1627}$ and international settings, ${ }^{1-578}$ and further confirm that both young and advanced maternal ages are risk factors for many important pregnancy outcomes. This paper complements these analyses by presenting year-by-year risks on the absolute scale in the US population, which enhances interpretability for clinicians and patients. The variability in form of risk curves for different outcomes (eg, progressive increase in risk for caesarean delivery compared with $\mathrm{u}$-shaped curve for SGA) suggests that the underlying mechanisms driving increased age-related risks may vary by outcome. Age is not a monolithic 'exposure' calling for a uniform clinical approach across outcomes. Nonetheless, our curves point to an optimal window for childbearing between ages 20 and 30, when nadirs occurred for most outcomes. Nearly half of Asian/Pacific Islander women and a quarter of white women begin childbearing after this window has passed, while a third of Black and Alaska Native/American Indian women begin childbearing before this window has begun.

The forms with which risks increase at maternal ages younger than 20 or older than 30 vary by maternal race. In general, risks of young childbearing were most pronounced for Asian/Pacific Islander and white women, for whom young childbearing was least common. Although risks were increased for all women for all outcomes after age 30, risks of delayed childbearing were more severe for Black and Alaska Native/American Indian women, for whom delayed childbearing was less common. The rapid elevation in risk with increasing age among Black women is especially important given the disproportionate burden of adverse pregnancy outcomes among Black women, most plausibly attributed to racism and related psychosocial stress. ${ }^{15} 16{ }^{28-30}$ On the other hand, risks increased more gradually with increasing maternal age for white and Asian/Pacific Islander women than for other women, though a larger proportion of first births to women in these groups are at older maternal age. Adjusting for covariates associated with maternal age and risks of adverse outcomes attenuated increased risks at increasing maternal ages, but not substantially.

In interpreting our findings, a number of limitations must be considered. Because we estimated risks at each maternal age based on risks to different women according to their age at first birth, differences in risk associated with each maternal age may be due to differences in women with a first birth at each age, rather than true biological risks of each outcome according to maternal age. Even after accounting for those covariates measured in these data, we could not examine the extent to which many potentially important characteristics (eg, pre-pregnancy health status, body mass index, smoking or infertility) varied by maternal age or race in this analysis, as these variables are not reliably collected in this database. ${ }^{1718} \mathrm{In}$ particular, infertility and subfertility, both strongly associated with maternal age ${ }^{31}$ and with increased risk for adverse outcomes, ${ }^{32}$ were not accounted for in our analyses. However, the unadjusted, population-average risks are most relevant for clinical practice, as clinical management is not usually 'adjusted' by age-related risk factors. As our study population was defined by birth during the study period, we could not examine risks of infertility, subfertility or miscarriage in this study, or whether these relationships vary by maternal race. Outcomes (particularly stillbirth) may be under-reported in these data, ${ }^{33}$ and this under-reporting may be most pronounced for American Indian/Alaska Native women. ${ }^{34}$ Although this study includes a national, population-based sample, findings may not be generalisable to settings with different 
distributions of characteristics that may alter relationships between maternal age and pregnancy outcomes. Furthermore, because the difference in baseline risks of adverse outcomes and the variability of age-outcome relationships according to maternal race in the USA are specific to the socioeconomic and sociopolitical contexts of this country, these relationships may differ in other settings.

As the observed associations may not be causal, recommendations regarding an optimal age range for conception among nulliparous women are beyond the scope of this paper. However, given the expected direction of confounders leading to pregnancies in women of young or advanced age, results reinforce the likelihood of an increased obstetric risk associated with delaying childbearing. Finally, in considering the optimal age for childbearing, sociocultural implications in terms of educational and career attainment $\mathrm{t}^{35}$ must be taken into account along with pregnancy risks, which we could not address in this study. Meanwhile, interventions to reduce adverse outcomes can target potential mediators by maternal age group, such as appropriate antenatal care in young women and prevention and control of chronic age-related disorders in older women.

\section{CONCLUSION}

Our analyses make a unique contribution to the literature regarding the relationship between maternal age at first birth and pregnancy and birth risks, and variability in these relationships by maternal race. Our findings confirm increased risk of adverse pregnancy and birth outcomes among first births to women of young and advanced maternal ages, provide easily interpretable risk curves and illuminate variability in these relationships across categories of maternal race in the USA.

\section{Author affiliations}

${ }^{1}$ Family Practice, Faculty of Medicine, The University of British Columbia, Vancouver, British Columbia, Canada

${ }^{2}$ Obstetrics and Gynecology, Beth Israel Deaconess Medical Center, Harvard Medical School, Boston, Massachusetts, USA

${ }^{3}$ Epidemiology, Harvard University T H Chan School of Public Health, Boston,

Massachusetts, USA

${ }^{4}$ Biostatistics, Harvard University T H Chan School of Public Health, Boston,

Massachusetts, USA

${ }^{5}$ Obstetrics and Gynaecology, University of British Columbia, Vancouver, British Columbia, Canada

${ }^{6}$ Obstetrics, Gynecology and Reproductive Biology, Brigham and Women's Hospital, Harvard Medical School, Boston, Massachusetts, USA

\section{Twitter Laura Schummers @DrLSchummers}

Acknowledgements The authors thank the National Vital Statistics System of the National Center for Health Statistics for maintaining the publicly available CohortLinked Infant Birth and Death files and Fetal Death files.

Contributors LS conducted all analyses and drafted all sections of the manuscript, as a component of her doctoral dissertation. LS, MRH, PLW JAH, TJV and SHD contributed to the conceptualisation of the paper. TFM contributed to the interpretation for clinical relevance. All authors provided input on the methodological approach and substantive relevance, contributed to data interpretation, revised multiple versions of the manuscript, approved the final version and are accountable for this work.
Funding LS was supported by a National Research Service Award from the Eunice Kennedy Shriver National Institute of Child Health and Human Development, National Institutes of Health (Doctoral Award; grant number 1F31HD086970-01A1), a Michael Smith Foundation for Health Research, Research Trainee Award (Postdoctoral Award; grant number 17934) and a Postdoctoral Health System Impact Fellowship cofunded by the BC Ministry of Health (grant number 18183) and the Canadian Institutes of Health Research (CIHR; grant number HI7-160383).

Competing interests None declared.

Patient consent for publication Not required.

Ethics approval This study received an exempt determination on the basis of not being human subjects research from the institutional review board of the Harvard School of Public Health (No 15-1891).

Provenance and peer review Not commissioned; externally peer reviewed.

Data availability statement Data are publicly available through the National Vital Statistics System of the National Center for Health Statistics, run by the US Centers for Disease Control and Prevention.

Open access This is an open access article distributed in accordance with the Creative Commons Attribution Non Commercial (CC BY-NC 4.0) license, which permits others to distribute, remix, adapt, build upon this work non-commercially, and license their derivative works on different terms, provided the original work is properly cited, appropriate credit is given, any changes made indicated, and the use is non-commercial. See: http://creativecommons.org/licenses/by-nc/4.0/.

ORCID iD

Laura Schummers http://orcid.org/0000-0003-3536-0498

\section{REFERENCES}

1 Lean SC, Derricott H, Jones RL, et al. Advanced maternal age and adverse pregnancy outcomes: a systematic review and metaanalysis. PLoS One 2017;12:e0186287.

2 Joseph KS, Allen AC, Dodds L, et al. The perinatal effects of delayed childbearing. Obstet Gynecol 2005;105:1410-8.

3 Laopaiboon M, Lumbiganon P, Intarut N, et al. Advanced maternal age and pregnancy outcomes: a multicountry assessment. Brit $J$ Obstet Gynaec 2014;121:49-56.

4 Klemetti R, Gissler M, Sainio S, et al. At what age does the risk for adverse maternal and infant outcomes increase? nationwide registerbased study on first births in Finland in 2005-2014. Acta Obstet Gyn Scand 2016;95:1368-75.

5 Waldenström U, Cnattingius S, Norman M, et al. Advanced maternal age and stillbirth risk in nulliparous and parous women. Obstet Gynecol 2015;126:355-62.

6 Reddy UM, Ko C-W, Willinger M. Maternal age and the risk of stillbirth throughout pregnancy in the United States. Am J Obstet Gynecol 2006;195:764-70.

7 Schummers L, Hutcheon JA, Hacker MR, et al. Absolute risks of adverse pregnancy and birth outcomes according to maternal age at first delivery: results from a population-based cohort. Epidemiology 2018;29:379-87.

8 Ganchimeg T, Ota E, Morisaki N, et al. Pregnancy and childbirth outcomes among adolescent mothers: a world Health organization multicountry study. BJOG: Int J Obstet Gy 2014;121:40-8.

9 Althabe F, Moore JL, Gibbons L, et al. Adverse maternal and perinatal outcomes in adolescent pregnancies: The Global Network's Maternal Newborn Health Registry study. Reprod Health 2015;12:S8.

10 Mathews TJ, Hamilton BE. Delayed childbearing: more women are having their first child later in life. NCHS Data Brief 2009;21.

11 Getahun D, Oyelese Y, Salihu HM, et al. Previous cesarean delivery and risks of placenta previa and placental abruption. Obstet Gynecol 2006;107:771-8.

12 Ghosh G, Grewal J, Männistö T, et al. Racial/Ethnic differences in pregnancy-related hypertensive disease in nulliparous women. Ethn Dis 2014;24:283-9.

13 DeFranco EA, Hall ES, Muglia LJ. Racial disparity in previable birth. Am J Obstet Gynecol 2016;214:e1-7.

14 Khalil A, Syngelaki A, Maiz N, et al. Maternal age and adverse pregnancy outcome: a cohort study. Ultrasound Obstet Gynecol 2013;42:634-43.

15 Gould JB, Madan A, Qin C, et al. Perinatal outcomes in two dissimilar immigrant populations in the United States: a dual epidemiologic paradox. Pediatrics 2003;111:e676-82.

16 Dominguez TP, Dunkel-Schetter C, Glynn LM, et al. Racial differences in birth outcomes: the role of general, pregnancy, and racism stress. Health Psychol 2008;27:194-203. 
17 Northam S, Knapp TR. The reliability and validity of birth certificates. Journal of Obstetric, Gynecologic \& Neonatal Nursing 2006;35:3-12.

18 Dietz P, Bombard J, Mulready-Ward C, et al. Validation of selected items on the 2003 U.S. standard certificate of live birth: new York City and Vermont. Pub Health Rep 2015;130:60-70.

19 Lydon-Rochelle MT, Holt VL, Cárdenas V, et al. The reporting of preexisting maternal medical conditions and complications of pregnancy on birth certificates and in hospital discharge data. Am J Obstet Gynecol 2005;193:125-34.

20 Villar J, Ismail LC, Victora CG, et al. International standards for newborn weight, length, and head circumference by gestational age and sex: the newborn cross-sectional study of the INTERGROWTH21st project. The Lancet 2014;384:857-68.

21 Kramer MS, Platt RW, Wen SW, et al. A new and improved population-based Canadian reference for birth weight for gestational age. Pediatrics 2001;108:e35-42.

22 Harrell FE. Regression modeling strategies with applications to linear models, logistic regression, and survival analysis. New York, NY: Springer, 2001.

23 Carpenter J, Bithell J. Bootstrap confidence intervals: when, which, what? A practical guide for medical statisticians. Stat Med 2000;19:1141-64.

24 Orsini N, Greenland S. A procedure to tabulate and plot results after flexible modeling of a quantitative covariate. Stata J 2011;11:1-29.

25 Altman DG, Bland JM. Statistics notes: interaction revisited: the difference between two estimates. BMJ 2003;326.
26 Corp S. Stata staistical software: release 14. College Station: Statacorp, LP, 2015

27 Cleary-Goldman J, Malone FD, Vidaver J, et al. Impact of maternal age on obstetric outcome. Obstet Gynecol 2005;105:983-90.

28 de Jonghe BE, Locke R, Paul DA, et al. The differential effects of maternal age, rae/ethnicity, and insurance on neonatal intensive care unit admission rates. BMC Preg Childbirth 2012;12:1-6.

29 Mustillo S, Krieger N, Gunderson EP, et al. Self-Reported experiences of racial discrimination and Black-White differences in preterm and low-birthweight deliveries: the cardia study. Am J Public Health 2004;94:2125-31.

30 Collins JW, David RJ, Handler A, et al. Very low birthweight in African American infants: the role of maternal exposure to interpersonal racial discrimination. Am J Public Health 2004;94:2132-8.

31 Sauer MV. Reproduction at an advanced maternal age and maternal health. Fertil Steril 2015;103:1136-43.

32 Allen VM, Wilson RD, Cheung A, et al. Pregnancy outcomes after assisted reproductive technology. J Obstet Gynaecol Can 2006;28:220-33.

33 Martin JA, Hoyert DL. The National fetal death file. Semin Perinatol 2002;26:3-11.

34 Wong CA, Gachupin FC, Holman RC, et al. American Indian and Alaska native infant and pediatric mortality, United States, 19992009. Am J Pub Health 2014;104:S320-8.

35 Hoffman S, Maynard R. Kids having kids: economic costs and social consequences of teen pregnancy. 2nd edn. Washington, DC: Urban Institute Press, 2008. 